Turk. J. Math. Comput. Sci.

12(2)(2020) 128-135

(C) MatDer

DOI : $10.47000 /$ tjmcs.691030

\title{
(Independent) $k$-Rainbow Domination of A Graph
}

\author{
Zhilla Mansouri $^{1}$ (D), Doost Ali Mojdeh $^{2, *}$ (D) \\ ${ }^{1}$ Department of Mathematics, University of Mazandaran, Babolsar, 47416-95447, Iran. \\ ${ }^{2}$ Department of Mathematics, University of Mazandaran, Babolsar, 47416-95447, Iran.
}

Received: 19-02-2020 • Accepted: 03-11-2020

Abstract. Let $G=(V, E)$ be a graph with the vertex set $V=V(G)$ and the edge set $E=E(G)$. Let $k$ be a positive integer and $\gamma_{r k}(G)\left(\gamma_{i_{r k}}(G)\right)$ be $k$-rainbow domination (independent $k$-rainbow domination) number of a graph $G$. In this paper, we study the $k$-rainbow domination and independent $k$-rainbow domination numbers of graphs. We obtain bounds for $\gamma_{r k}(G-e)\left(\gamma_{i_{r k}}(G-e)\right)$ in terms of $\gamma_{r k}(G)\left(\gamma_{i_{r k}}(G)\right)$. Finally, the relation between weak 3-domination and 3-rainbow domination number of graphs will be investigated.

2010 AMS Classification: 05 C69

Keywords: Rainbow domination, Independent rainbow domination, Graphs.

\section{INTRODUCTION}

Let $G=(V, E)$ be a simple finite graph with the vertex set $V=V(G)$ and the edge set $E=E(G)$. For the terminologies and notations which are not defined here explicitly, we may use [9] as a reference. The order of $G$ is the number of vertices of $G$. For any vertex $v \in V$, the open neighborhood of $v$ is $N(v)=\{u \in V \mid u v \in E\}$ and its closed neighborhood is $N[v]=N(v) \cup\{v\}$. For a set $S \subseteq V$, the open neighborhood of $S$ is $N(S)=\bigcup_{v \in S} N(v)$ and its closed neighborhood is $N[S]=N(S) \cup S$.

A set $S \subseteq V$ is a dominating set if every vertex in $V \backslash S$ is adjacent to at least one vertex in $S$. The domination number $\gamma(G)$ is the minimum cardinality of a dominating set in $G$. A dominating set with cardinality $\gamma(G)$ is called a $\gamma(G)$-set.

In 2008, Bresar et al. [2] introduced the k-rainbow domination as a generalization of domination in graphs.

Definition 1.1. ( [2]) Let $k$ be a positive integer, $[k]=\{1,2, \ldots, k\}$ and $\mathcal{P}([k])$ be the power set of $[k]$. For any graph $G$, a function $f: V(G) \rightarrow \mathcal{P}([k])$ is a $k$-rainbow dominating function (or $k$-rD function for short) if for every vertex $v \in V$ with $f(v)=\varnothing, f(N(v))=\bigcup_{u \in N(v)} f(u)=[k]$. The weight $w(f)$ of a $k$-rD function $f$ is defined as $w(f)=\sum_{v \in V(G)}|f(v)|$. The minimum weight of a $k$-rDF of $G$ is called the $k$-rainbow domination number of $G$ and is denoted by $\gamma_{r k}(G)$.

A $k$-rDF $f$ is an independent $k$-rainbow dominating function (I $k$-rD function) if no two vertices assigned nonempty sets are adjacent. The weight of an $\mathrm{I} k$-rD function $f$ is $w(f)=\sum_{v \in V(G)}|f(v)|$. The independent k-rainbow domination number $\gamma_{i_{r k}}(G)$ is the minimum weight of an I $k$-rDF of $G$.

*Corresponding Author

Email addresses: mansoury.zh@yahoo.com (Zh. Mansouri), damojdeh@umz.ac.ir (D.A. Mojdeh) 
Chang et al. [4] were quick on the uptake and showed that, for positive integer $k$, the $k$-rainbow domination problem is $N P$-complete even when restricted to chordal graphs and bipartite graphs. The same paper shows that there is a lineartime algorithm to determine the parameter for trees. The paper also shows that the problem remains $N P$-complete for planar graphs.

Notice that the above discussion shows that $\gamma_{r k}(G)$ is a non-decreasing function in $k$. Moreover, Chang et al. [4] showed that for all graphs $G$ on $n$ vertices and all positive integer $k, \min \{k, n\} \leq \gamma_{r k}(G) \leq n$.

Many other papers establish bounds on this parameter and investigate the 2-rainbow domination number versus the total domination number and the (weak) Roman domination number. Also, the edge or vertex critical graphs with respect to the rainbow domination have been investigated in literature. For reading the results for special families of graphs such as paths, cycles and the generalized Petersen graphs the reader can consult $[1,2,4,10]$. The rainbow domination numbers are studied for digraphs and also Cartesian product of some digraphs [5]. Also outer independent rainbow dominating functions of graphs has been studied [6].

Pai and Chiu [8] developed an exact algorithm and a heuristic for 3-rainbow domination. Recently, Chang et al. showed that the $k$-rainbow domination number is equal to the so-called weak $k$-domination number for strongly chordal graphs (see [2,3]).

A linear algorithm for determining a 2-rD function of minimum weight of an arbitrary tree was presented in [2]. The algorithm was based on the related concept of so-called weak 2-domination. Intuitively, we could call it a monochromatic version of 2-rainbow domination.

Let $G=(V, E)$ be a graph and $f: V(G) \rightarrow\{0,1,2\}$ be a function that assigns to each vertex a number chosen from $\{0,1,2\}$. For notational convenience, we define

$$
f[v]=\sum_{u \in N[v]} f(u)
$$

for each $v \in V$. We call $v \in V$ a bad vertex with respect to $f$ if $f(v)=0$ and $f[v] \leq 1$; otherwise, we say that $v$ is a good vertex with respect to $f$. Note that if $v$ is a good vertex with respect to $f$ and $f(v)=0$, then $f[v] \geq 2$. If every vertex of $T$ is a good vertex with respect to $f$, then $f$ is called a weak \{2\}-dominating function (W2D function) of $G$. The weight $w(f)$ of $f$ is defined as $w(f)=\sum_{v \in V} f(v)$. The minimum weight of a W2D function in $G$ is called the weak $\{2\}$-domination number of $G$, denoted by $\gamma_{w 2}(G)$.

\section{2. $k$-RAINBOW AND INDEPENDENT $k$-RAINBOW DOMINATION OF SOME SPECIAL GRAPHS}

The rainbow domination numbers of some families of graphs has been already known. In this section, we study the (independent) rainbow domination numbers of some other families of graphs, for example Harary graphs, complete and complete $r(\geq 2)$-partite graphs, paths and cycles.

The domination parameters of Harary graphs have been studied in [7]. Here we study the 2-rainbow domination number of Harary graphs. Given the positive integers $k<n$, place $n$ vertices around a circle, equally spaced. If $k$ is even, form $H_{k, n}$ by making each vertex adjacent to the nearest $k / 2$ vertices in each direction around the circle. If $k$ is odd and $n$ is even, form $H_{k, n}$ by making each vertex adjacent to the nearest $(k-1) / 2$ vertices in each direction and to the diametrically opposite vertex. In each case, $H_{k, n}$ is $k$-regular. When $k$ and $n$ are both odd, index the vertices by the integers modulo $n$. Construct $H_{k, n}$, from $H_{k-1, n}$ by adding the edges $i \leftrightarrow i+(n-1) / 2$ for $0 \leq i \leq(n-1) / 2$.

Lemma 2.1. Let $H_{k, n}(2 \leq k<n)$ be a Harary graph.

(i) If $k$ is an even integer and $n=q(k+2)+r, 0 \leq r \leq k+1$, then

$$
\gamma_{r 2}\left(H_{k, n}\right)= \begin{cases}2 q & \text { if } r=0, \\ 2 q+1 & \text { if } r=1, \\ 2(q+1) & \text { if } 2 \leq r \leq k+1 .\end{cases}
$$

(ii) If $k$ is an odd integer and $n=q(k+1)+r, 0 \leq r \leq k$, then

$$
\gamma_{r 2}\left(H_{k, n}\right)= \begin{cases}2 q & \text { if } r=0, \\ 2 q+1 & \text { if } r=1, \\ 2(q+1) & \text { if } 2 \leq r \leq k+1 .\end{cases}
$$


Proof. (i) Let $k$ be even. First we show that for any $k+2$ consecutive vertices

$$
v_{i_{1}}, v_{i_{2}}, \ldots, v_{i_{(k / 2)+1}}, v_{i_{(k / 2)+2}}, \ldots, v_{i_{k+1}}, v_{i_{k+2}},
$$

there exist at least two vertices $v_{i_{l}}$ and $v_{i_{l}}$ with $f\left(v_{i_{l}}\right) \cup f\left(v_{i_{l}}\right)=\{1,2\}$ or there exists one vertex $v_{i_{l}}$ with $f\left(v_{i_{l}}\right)=\{1,2\}$. Suppose to the contrary that there exists only one vertex like $v_{i_{l}}$ with value $\{1\}$ or $\{2\}$, then we cannot assign any value to $v_{i_{l+(k / 2)+1}}$ or $v_{i_{l-(k / 2)-1}}$, a contradiction.

Now we give a 2-rainbow dominating function of $H_{k, n}$ as follows: Let $n=q(k+2)$ and $V\left(H_{k, n}\right)=\left\{v_{1}, \ldots, v_{(k / 2)+2}, \ldots, v_{k+3}, \ldots, v_{q(k+2)}\right\}$. We define $f: V\left(H_{k, n}\right) \rightarrow \mathcal{P}(\{1,2\})$ by

$$
f\left(v_{m((k / 2)+1)+1}\right)= \begin{cases}\{1\} & \text { if } 0 \leq m \leq 2 q-1 \text { is even, } \\ \{2\} & \text { if } 0 \leq m \leq 2 q-1 \text { is odd, } \\ \varnothing & \text { otherwise. }\end{cases}
$$

Let $n=q(k+2)+1$ and $V\left(H_{k, n}\right)=\left\{v_{1}, \ldots, v_{(k / 2)+2}, \ldots, v_{k+3}, \ldots, v_{q(k+2)}, v_{q(k+2)+1}\right\}$. We define $f: V\left(H_{k, n}\right) \rightarrow \mathcal{P}(\{1,2\})$ by

$$
f\left(v_{m\left(\frac{k}{2}+1\right)+1}\right)= \begin{cases}\{1\} & \text { if } 0 \leq m \leq 2 q \text { is even } \\ \{2\} & \text { if } 0 \leq m \leq 2 q-1 \text { is odd, } \\ \varnothing & \text { otherwise. }\end{cases}
$$

Let $n=q(k+2)+r$, where $2 \leq r \leq k+1$ and

$$
V\left(H_{k, n}\right)=\left\{v_{1}, \ldots, v_{(k / 2)+2}, \ldots, v_{k+3}, \ldots, v_{q(k+2)}, v_{q(k+2)+1}, \ldots, v_{q(k+2)+r}\right\} .
$$

We define $f: V\left(H_{k, n}\right) \rightarrow \mathcal{P}(\{1,2\})$ by $f\left(v_{q(k+2)+1+\lfloor r / 2\rfloor}\right)=\{2\}$,

$$
f\left(v_{m((k / 2)+1)+1}\right)= \begin{cases}\{1\} & \text { if } 0 \leq m \leq 2 q \text { is even, } \\ \{2\} & \text { if } 0 \leq m \leq 2 q-1 \text { is odd }\end{cases}
$$

and $f\left(v_{i}\right)=\varnothing$ for otherwise. Therefore we have the desired formula.

(ii) Let $k$ be odd. We can use a method similar to that of (i) to establish the result.

The $k$-rainbow domination numbers of the paths, cycles and generalized Petersen graphs have already been considered elsewhere. In this section, we study the independent 3-rainbow domination number of paths and the independent 2, 3-rainbow domination numbers of cycles and provide a construction for the I3-rD function with the desired weight in each case.

The independent 2-rainbow domination number of trees has been studied in [1] and in particular, it has been shown that, for any path $P_{n}, \gamma_{i_{r 2}}\left(P_{n}\right)=\left\lfloor\frac{n}{2}\right\rfloor+1$.

Proposition 2.2.

$$
\gamma_{i_{r 3}}\left(P_{n}\right)= \begin{cases}\left\lceil\frac{3 n+1}{4}\right\rceil & \text { if } n=0 \text { or } 1(\bmod 4), \\ \left\lceil\frac{3 n+3}{4}\right\rceil & \text { if } n=2 \text { or } 3(\bmod 4) .\end{cases}
$$

Proof. Let $v_{1}, \cdots, v_{n}$ be the vertices of $P_{n}$. First of all, without loss of generality, by $\left|f\left(v_{i}\right)\right|=0,\left|f\left(v_{i}\right)\right|=1,\left|f\left(v_{j}\right)\right|=2$ and $\left|f\left(v_{k}\right)\right|=3$ we mean that $f\left(v_{i}\right)=\varnothing, f\left(v_{i}\right)=\{1\}, f\left(v_{j}\right)=\{2,3\}$ and $f\left(v_{k}\right)=\{1,2,3\}$, respectively. It is well known that if for a vertex $v_{i}, f\left(v_{i}\right)=\varnothing$, then for the independent 3-rainbow domination one of the following must be held:

(i) $\left|f\left(v_{i-1}\right)\right|=3$ or $\left|f\left(v_{i+1}\right)\right|=3$, (ii) $\left|f\left(v_{i-1}\right)\right|=1$ and $\left|f\left(v_{i+1}\right)\right|=2$, or (iii) $\left|f\left(v_{i-1}\right)\right|=2$ and $\left|f\left(v_{i+1}\right)\right|=1$.

We now prove the result by induction on $n$. It is clear for $n \in\{1,2,3\}$. For $n \in\{4,5,6,7\}$ it is easy to see that $\gamma_{i_{r 3} 3}\left(P_{4}\right)=4=\left\lceil\frac{3 n+1}{4}\right\rceil$ by assigning $\left|f\left(v_{1}\right)\right|=1,\left|f\left(v_{3}\right)\right|=3$ and $\left|f\left(v_{i}\right)\right|=0$ for $i=2,4 ; \gamma_{i_{3}}\left(P_{5}\right)=4=\left\lceil\frac{3 n+1}{4}\right\rceil$ by assigning $\left|f\left(v_{1}\right)\right|=1=\left|f\left(v_{5}\right)\right|,\left|f\left(v_{3}\right)\right|=2$ and $\left|f\left(v_{i}\right)\right|=0$ for $i=2,4 ; \gamma_{i_{3}}\left(P_{6}\right)=6=\left\lceil\frac{3 n+3}{4}\right\rceil$ by assigning $\left|f\left(v_{1}\right)\right|=1,\left|f\left(v_{3}\right)\right|=2$, $\left|f\left(v_{5}\right)\right|=3$ and $\left|f\left(v_{i}\right)\right|=0$ for $i=2,4,6 ; \gamma_{i_{3} 3}\left(P_{7}\right)=6=\left\lceil\frac{3 n+3}{4}\right\rceil$ by assigning $\left|f\left(v_{1}\right)\right|=1,\left|f\left(v_{3}\right)\right|=2,\left|f\left(v_{5}\right)\right|=1$, $\left|f\left(v_{7}\right)\right|=2$ and $\left|f\left(v_{i}\right)\right|=0$ for $i=2,4,6$. Hence the basis of induction holds. Let $n=4 t, t \geq 2$ and the result holds for $n=4 t-4$ by assigning $\left|f\left(v_{i}\right)\right|=1$ for $i \equiv 1(\bmod 4),\left|f\left(v_{i}\right)\right|=2$ for $i \equiv 3(\bmod 4)$ and $i \neq 4 t-5,\left|f\left(v_{4 t-5}\right)\right|=3$ and $\left|f\left(v_{i}\right)\right|=0$ for otherwise. Hence $\gamma_{i_{r_{3}}}\left(P_{4 t-4}\right)=\left\lceil\frac{3(4 t-4)+1}{4}\right\rceil$. For $n=4 t, t \geq 2$, by using induction, we assign the vertex $v_{k}$ with $k \leq 4 t-6$ same as induction, and for the vertices $v_{4 t-5}-v_{4 t}$, we consider $\left|f\left(v_{4 t-5}\right)\right|=2, f\left(v_{4 t-3}\right)\left|=1, f\left(v_{4 t-4}\right)\right|=3$ and $f\left(v_{4 t-4}\right)\left|=f\left(v_{4 t-2}\right)\right|=f\left(v_{4 t}\right) \mid=0$. Therefore $\gamma_{i_{r 3}}\left(P_{4 t}\right)=\gamma_{i_{r 3}}\left(P_{4 t-4}\right)+3=\left\lceil\frac{3(4 t-4)+1}{4}\right\rceil+3=\left\lceil\frac{3(4 t-4)+1+12}{4}\right\rceil=\left\lceil\frac{3 n+1}{4}\right\rceil$. For $n \equiv 1,2,3(\bmod 4)$, the proofs are similar. 
We have the exact formulas for $\gamma_{i_{r 2}}\left(P_{n}\right)$ and $\gamma_{i_{r 3}}\left(P_{n}\right)$. In what follows, we give the exact formula for $\gamma_{i_{r k}}\left(P_{n}\right)$ for $k \geq 4$. We have $\gamma_{i_{r k}}\left(P_{1}\right)=1, \gamma_{i_{r k}}\left(P_{2}\right)=\gamma_{i_{r k}}\left(P_{3}\right)=k$. So, we may assume that $n \geq 4$.

Proposition 2.3. For $n, k \geq 4$,

$$
\gamma_{i_{r k}}\left(P_{n}\right)= \begin{cases}k t+1 & \text { if } n=4 \text { tor } 4 t+1, \\ k(t+1) & \text { otherwise. }\end{cases}
$$

Proof. Let $f: V\left(P_{n}\right) \rightarrow \mathcal{P}(\{1,2, \cdots, k\})$ be a $\gamma_{i_{r k}}\left(P_{n}\right)$-function. Let $P_{n}$ be a path with vertices $v_{1}, \cdots, v_{n}$. We consider four cases.

Case 1. $n=4 t$. It is easy to see that $w\left(f\left(P_{4}\right)\right)=k+1$. Consider the subpaths $P_{i}: v_{4 i-3} v_{4 i-2} v_{4 i-1} v_{4 i}$, for $1 \leq i \leq t$. It is straightforward to see that $w\left(\left.f\right|_{P_{i}}\right) \geq k$, for all $1 \leq i \leq t$. Therefore, $\gamma_{i_{r k}}\left(P_{n}\right)=w(f) \geq k t$. Suppose now that $w(f)=k t$. Therefore, $w\left(\left.f\right|_{P_{i}}\right)=k$ for all $1 \leq i \leq t$. Now if three vertices of a subpath $P_{i}$ have the weight $\varnothing$ under $f$, then $w\left(\left.f\right|_{P_{i-1}}\right)>k$ or $w\left(\left.f\right|_{P_{i+1}}\right)>k$. This is a contradiction. So, exactly two vertices of $P_{i}$ have the weight $\varnothing$ under $f$. We now show that $\left|f\left(v_{4 i}\right)\right|=0$ for $1 \leq i \leq t-1$ where $t \geq 2$. It is clear that $\left|f\left(v_{4}\right)\right|=0$. Suppose that $\left|f\left(v_{4 i}\right)\right|=0$ for $1 \leq i \leq t-2$. If $\left|f\left(v_{4 i+1}\right)\right|=0$, then $\left|f\left(v_{4 i+2}\right)\right|=k$ and $\left|f\left(v_{4(i+1)}\right)\right| \geq 1$, a contradiction. Thus $\left|f\left(v_{4 i+1}\right)\right|=[j],\left|f\left(v_{4 i+2}\right)\right|=0$, $\left|f\left(v_{4 i+3}\right)\right|=[k] \backslash[j]$ and $\left|f\left(v_{4(i+1)}\right)\right|=0$. Now we clime that $\left|f\left(\bigcup_{i=4 t-3}^{4 t}\left\{v_{i}\right\}\right)\right| \geq k+1$. Because if $\left|f\left(v_{4 t-3}\right)\right|=0$, then $\left|f\left(v_{4 t-2}\right)\right|=k$ and $\left|f\left(v_{4 t-1}\right) \cup f\left(v_{4 t}\right)\right| \geq 1$, and if $\left|f\left(v_{4 t-3}\right)\right| \geq 1$, then $\left|f\left(v_{4 t-2}\right) \cup f\left(v_{4 t-1}\right) \cup f\left(v_{4 t}\right)\right| \geq k$. Therefore $\gamma_{i_{r k}}\left(P_{n}\right)=w(f) \geq k t+1$ for $n=4 t$.

On the other hand, we give an $\mathrm{I} k$-rD function $g$ with $w(g)=k t+1$. Let $g: V\left(P_{n}\right) \rightarrow \mathcal{P}(\{1,2, \cdots, k\})$ be defined by

$$
g(v)= \begin{cases}\varnothing & v=v_{4 i-2}, v_{4 i}(1 \leq i \leq t) \\ \{1\} & v=v_{4 i-3}(1 \leq i \leq t) \\ \{2, \cdots, k\} & v=4 i-1(1 \leq i \leq t) \\ \{1, \cdots, k\} & v=4 t-1\end{cases}
$$

Then, $g$ is an I $k$-rDF of $P_{n}$ with weight $k t+1$. So, $\gamma_{i_{r k}}\left(P_{n}\right) \leq k t+1$. This shows that $\gamma_{i_{r k}}\left(P_{n}\right)=k t+1$.

Case 2. $n=4 t+1$. Similar to the Case 1, we can show that $w(f) \geq k t+1$. Now the function $g^{\prime}: V\left(P_{n}\right) \rightarrow$ $\mathcal{P}(\{1,2, \cdots, k\})$ defined by

$$
g^{\prime}(v)= \begin{cases}\{1\} & v=v_{4 i-3}(1 \leq i \leq t), \\ \varnothing & v=v_{4 i-2}, v_{4 i}(1 \leq i \leq t), \\ \{2, \cdots, k\} & v=v_{4 i-1}(1 \leq i \leq t),\end{cases}
$$

is an $\mathrm{I} k$-rDF of $P_{n}$ with weight $k t+1$. This shows that $\gamma_{i_{r k}}\left(P_{n}\right)=k t+1$.

Case 3. $n=4 t+2$. Let $n=6$. By assigning [1] to $v_{1},[k] \backslash[1]$ to $v_{3}$, [k] to $v_{5}$ and $\varnothing$ to the vertices $v_{2}, v_{4}, v_{6}$. Then we have $\gamma_{i_{r k}}\left(P_{6}\right)=2 k$. Suppose now that $n=4 t+2$, where $t \geq 2$. We have $P_{4 t}=P_{n}-\left\{v_{4 t+1}, v_{4 t+2}\right\}$. Using Case 1 , it has been seen $w\left(\left.f\right|_{P_{4 t}}\right) \geq k t$ provided that $f\left(v_{4 t-1}\right)=[k-1]$. In this case, we should assign $f\left(v_{4 t+1}\right)=[k]$ and $f\left(v_{4 t+2}\right)=\varnothing$ in $P_{n}$. Thus $\gamma_{i_{r k}}\left(P_{n}\right) \geq k(t+1)$.

Now the function $h: V\left(P_{n}\right) \rightarrow \mathcal{P}(\{1,2, \cdots, k\})$ defined by

$$
h(v)= \begin{cases}\{1\} & v=v_{4 i-3}(1 \leq i \leq t), \\ \varnothing & v=v_{4 i-2}(1 \leq i \leq t+1) \text { and } v=v_{4 i}(1 \leq i \leq t), \\ \{2, \cdots, k\} & v=v_{4 i-1}(1 \leq i \leq t), \\ \{1,2, \ldots, k\} & v=v_{4 t+1},\end{cases}
$$

is an $\mathrm{I} k$-rDF of $P_{n}$ with weight $k(t+1)$. This shows that $\gamma_{i_{r k}}\left(P_{n}\right)=k(t+1)$.

Case 4. $n=4 t+3$. We have $w\left(\left.f\right|_{P_{i}}\right) \geq k$, for all $1 \leq i \leq t$. So by Case $3, \gamma_{i_{r k}}\left(P_{n}\right)=w(f) \geq k(t+1)$.

We now define the function $h^{\prime}: V\left(P_{n}\right) \rightarrow \mathcal{P}(\{1,2, \cdots, k\})$ by

$$
h^{\prime}(v)= \begin{cases}\{1\} & v=v_{4 i-3}, \\ \varnothing & v=v_{m} \text { for even positive integer } m, \\ \{2, \cdots, k\} & v=4 i-1(1 \leq i \leq t+1) .\end{cases}
$$

It is easy to see that $h^{\prime}$ is an I $k$-rDF of $P_{n}$ with weight $k(t+1)$. So, $\gamma_{i_{r k}}\left(P_{n}\right)=k(t+1)$. This completes the proof. 
The 2-rainbow domination of a cycle has been studied in [3].

Proposition 2.4. ( [3], Proposition 3.2) For $n \geq 3, \gamma_{r 2}\left(C_{n}\right)=\left\lfloor\frac{n}{2}\right\rfloor+\left\lceil\frac{n}{4}\right\rceil-\left\lfloor\frac{n}{4}\right\rfloor$.

The independent $k$-rainbow domination of a cycle is studied here and we known that $\gamma_{r 2}(G) \leq \gamma_{i_{r 2}}(G)$. For the cycle $C_{3}$ it is easy to see $\gamma_{i_{r 2}}\left(C_{3}\right)=2$. For $\gamma_{i_{r 2}}\left(C_{n}\right)$ we have the following.

Proposition 2.5. For $n \geq 4, \gamma_{i_{r 2}}\left(C_{n}\right)= \begin{cases}\frac{n}{2} & \text { if } n=4 k, \\ \left\lceil\frac{n}{2}\right\rceil+1 & \text { otherwise. }\end{cases}$

Proof. For any cycle $C_{n}$, Proposition 2.4 implies that $\gamma_{i_{r 2}}\left(C_{n}\right) \geq\left\lfloor\frac{n}{2}\right\rfloor+\left\lceil\frac{n}{4}\right\rceil-\left\lfloor\frac{n}{4}\right\rfloor$. Let $n \equiv 0(\bmod 4)$. By assigning $\{1\}$ to $v_{4 i+1},\{2\}$ to $v_{4 i+3}$ for $0 \leq i \leq \frac{n}{4}-1$ and $\varnothing$ to the other vertices, we deduce that $\gamma_{i_{r 2}}\left(C_{n}\right)=\frac{n}{2}$ for $n \equiv 0(\bmod 4)$.

Let $n \equiv 2(\bmod 4)$. By assigning $\{1,2\}$ to $v_{1},\{1\}$ to $v_{4 i+1}$ for $1 \leq i \leq \frac{n-2}{4}$, value $\{2\}$ to $v_{4 i+3}$ for $0 \leq i \leq \frac{n-6}{4}$, and $\varnothing$ to the other vertices, we have $\gamma_{i_{r 2}}\left(C_{n}\right)=\left\lceil\frac{n}{2}\right\rceil+1$ for $n \equiv 2(\bmod 4)$.

Let $n$ be odd and let $f$ be an I2-rD function of $C_{n}$ of minimum weight. There is a vertex $x \in V\left(C_{n}\right)$ with $f(x)=\{1,2\}$. Then we get $w(f) \geq 2+\gamma_{i_{r 2}}\left(P_{n-3}\right)=2+\left\lfloor\frac{n-3}{2}\right\rfloor+1=2+\frac{n-3}{2}+1=\frac{n+1}{2}+1=\left\lceil\frac{n}{2}\right\rceil+1$.

The independent 3-rainbow domination number of cycle $C_{3}$ is 3 . In the follows, we establish the independent 3-rainbow domination number of any cycle of order $n \geq 4$.

Proposition 2.6. For $n \geq 4, \gamma_{i_{r} 3}\left(C_{n}\right)= \begin{cases}3\left\lceil\frac{n}{4}\right\rceil+1 & \text { if } n=4 k+3, \\ 3\left\lceil\frac{n}{4}\right\rceil & \text { otherwise. }\end{cases}$

Proof. Let $n \equiv 0(\bmod 4)$. It is clear that $\gamma_{i_{r 3}}\left(C_{n}\right) \leq \frac{3 n}{4}$. Let $f$ be an I3-rD function with minimum weight. There is a vertex $v_{i}$ with $f\left(v_{i}\right)=0$. Let $P_{n-1}=C_{n}-\left\{v_{i}\right\}$. Then $\gamma_{i_{r 3}}\left(C_{n}\right) \geq \gamma_{i_{r 3}}\left(P_{n-1}\right)=\left\lceil\frac{3(n-1)+3}{4}\right\rceil=\frac{3 n}{4}$ by Proposition 2.2 . Therefore, $\gamma_{i_{r} 3}\left(C_{n}\right)=\frac{3 n}{4}$ for $n \equiv 0(\bmod 4)$.

Let $n \equiv 1(\bmod 4)$. Then $\gamma_{i_{r}}\left(C_{n}\right) \leq 3\left\lceil\frac{n}{4}\right\rceil$. Let $f$ be an I3-rD function with minimum weight on $C_{n}$. There is a vertex $v_{i}$ with $f\left(v_{i}\right)=3, f\left(v_{i-1}\right)=f\left(v_{i+1}=\varnothing\right.$. Let $P_{n-3}=C_{n}-\left\{v_{i-1}, v_{i}, v_{i+1}\right\}$. Then we get $w(f) \geq 3+\gamma_{i_{r 3}}\left(P_{n-3}\right)=3+\left\lceil\frac{3 n-6}{4}\right\rceil=$ $\left\lceil\frac{3 n+3}{4}\right\rceil=3\left\lceil\frac{n}{4}\right\rceil$, by proposition 2.2. Therefore, the result holds. The other two parts can be proved similarly.

We have already obtained the exact values for $\gamma_{i_{r 2}}\left(C_{n}\right), \gamma_{i_{r 3}}\left(C_{n}\right)$ and $\gamma_{i_{r k}}\left(P_{n}\right)$. In what follows, we give the exact value for $\gamma_{i_{r k}}\left(C_{n}\right)$ for $k \geq 4$. It is easy to see that $\gamma_{i_{r k}}\left(C_{3}\right)=k=\gamma_{i_{r k}}\left(C_{4}\right)$ and $\gamma_{i_{r k}}\left(C_{5}\right)=2 k=\gamma_{i_{r k}}\left(C_{6}\right)$. In general, we have the following.

Proposition 2.7. For $n \geq 7$ and $k \geq 4$,

$$
\gamma_{i_{r k}}\left(C_{n}\right)= \begin{cases}k t & \text { if } n=4 t \\ k(t+1) & \text { if } n=4 t+2 \text { or } n=4 t+1, \\ k(t+1)+1 & \text { if } n=4 t+3\end{cases}
$$

Proof. Let $n=4 t$. Since at least $2 t$ vertices should be assigned by $\varnothing$ and any such vertices must be adjacent to vertices with weight at least $[k]$, any $\mathrm{I} k$-rD function $f$ with $f\left(v_{4 i-3}\right)=\{1\}, f\left(v_{4 i-1}\right)=\{2, \ldots, k\}$, and $f\left(v_{4 i-2}\right)=f\left(v_{4 i}\right)=\varnothing$ for $1 \leq i \leq t$ is a $\gamma_{i_{r k}}\left(C_{4 t}\right)$-function. Therefore $\gamma_{i_{r k}}\left(C_{4 t}\right)=k t$.

Let $n=4 t+2$. By deleting three vertices $v_{n}, v_{n-1}, v_{n-2}$ from $C_{n}$ we will have a path $P_{4 t-1}$. By Proposition 2.3, $\gamma_{i_{r k}}\left(P_{4 t-1}\right)=k t$. Two vertices $v_{n}$ and $v_{n-2}$ of these three vertices should be assigned by $\varnothing$ and the vertex $v_{n-1}$ by $[k]$. Therefore, $\gamma_{i_{r k}}\left(P_{4 t+2}\right)=k(t+1)$.

Let $n \geq 7$ be an odd integer. Then for any $\mathrm{I} k$-rD function $f$, there are two consecutive vertices which will be assigned $\varnothing$ under $f$. If these two vertices are $v_{i}$ and $v_{i+1}$, then without lose of generality we should assign $f\left(v_{i-1}\right)=f\left(v_{i+2}\right)=[k]$ and $f\left(v_{i-2}\right)=f\left(v_{i+3}\right)=\varnothing$. Thus these six vertices have weight $2 k$.

Now let $n=4 t+1$. By deleting these consecutive six vertices from $C_{n}$ we obtain a path $P_{4(t-2)+3}$. By Proposition 2.3, $\gamma_{i_{r k}}\left(P_{4(t-2)+3}\right)=k(t-1)$. Therefore $\gamma_{i_{r k}}\left(C_{4 t+1}\right)=k(t+1)$.

Let $n=4 t+3$. By deleting these consecutive six vertices from $C_{n}$, we obtain a path $P_{4(t-1)+1}$. By Proposition 2.3, $\gamma_{i_{r k}}\left(P_{4(t-1)+1}\right)=k(t-1)+1$. Therefore $\gamma_{i_{r k}}\left(C_{4 t+3}\right)=k(t+1)+1$. 


\section{3. $k$-Rainbow and IndePEndent $k$-Rainbow Domination of $G-e$ For Any Graph $G$}

We shall study the effect of removing an edge on the $k$-Rainbow domination and independent $k$-Rainbow domination numbers of a graph $G$.

Proposition 3.1. Let $G$ be a graph. Then

$$
\gamma_{r k}(G) \leq \gamma_{r k}(G-e) \leq \gamma_{r k}(G)+1
$$

This bounds are sharp.

Proof. Let $e=x y$ be an edge of $G$. Let $G^{\prime}=G-e$ and $f: V\left(G^{\prime}\right) \rightarrow \mathcal{P}(\{1,2, \ldots, k\})$ be a $k$-rD function with minimum weight of $G^{\prime}$. It is easy to see that $f$ is a $k$-rD function of $G$, as well. Therefore, $\gamma_{r k}(G) \leq \sum_{v \in V(G)}|f(v)|=\gamma_{r k}\left(G^{\prime}\right)$.

Suppose now that $g: V(G) \rightarrow \mathcal{P}(\{1,2, \ldots, k\})$ is a $k$-rD function of $G$ of the minimum weight. If $g(x)=g(y)=\varnothing$ or $g(x), g(y) \neq \varnothing$, then $g$ is a $k$-rD function of $G^{\prime}$, as well. So, $\gamma_{r k}\left(G^{\prime}\right) \leq \sum_{v \in V\left(G^{\prime}\right)}|g(v)|=\gamma_{r k}(G)$. Without loss of generality, we suppose that $g(x)=\varnothing$ and $g(y) \neq \varnothing$. Then, $h: V\left(G^{\prime}\right) \rightarrow \mathcal{P}(\{1,2, \ldots, k\})$ defined by

$$
h(v)= \begin{cases}\{1\} & \text { if } v=x \\ g(v) & \text { otherwise }\end{cases}
$$

is a $k$-rD function of $G$. Therefore,

$$
\gamma_{r k}\left(G^{\prime}\right) \leq \sum_{v \in V\left(G^{\prime}\right)}|h(v)|=\sum_{v \in V(G)}|g(v)|+1=\gamma_{r k}(G)+1 .
$$

To see the sharpness of lower bound, let $G=K_{n}$. Then $\gamma_{r k}(G)=\gamma_{r k}(G-e)$. For sharpness of the upper bound, let $G=S_{n}$ be a star graph. Then $\gamma_{r k}(G-e)=k+1=\gamma_{r k}(G)+1$ for $n \geq k+1$. This completes the proof.

We establish here the independent $k$-rainbow domination numbers of subgraph $G-e$ of $G$.

Proposition 3.2. Let $G$ be a graph and e be an edge of the $G$. Then,

$$
\gamma_{i_{r k}}(G-e) \leq \gamma_{i_{r k}}(G)+k-1 \text {. }
$$

Proof. Let $e=x y$ and $f: V(G) \rightarrow \mathcal{P}(\{1,2, \ldots, k\})$ be a $\gamma_{i_{r k}}(G)$-function. We may assume that $f(y)=\varnothing$. If $f(x)=\varnothing$, then $f$ is an $\mathrm{I} k$-rD function of $G-e$. So, $\gamma_{i_{r k}}(G-e) \leq \sum_{v \in V(G)}|f(v)|=\gamma_{i_{r k}}(G)$. We now let $f(x) \neq \varnothing$. We distinguish two cases depending on $f(x)$.

Case 1. There exists a vertex $z \in N_{G}(y)-\{x\}$ with nonempty weight. We define $f^{\prime}: V(G-e) \rightarrow \mathcal{P}(\{1,2, \ldots, k\})$ by

$$
f^{\prime}(v)= \begin{cases}\{1,2, \ldots, k\} & \text { if } v=z \\ f(v) & \text { if } v \neq z\end{cases}
$$

It is easy to see that $f^{\prime}$ is an $\mathrm{I} 2-\mathrm{rD}$ function of $G-e$. Therefore,

$$
\gamma_{i_{r k}}(G-e) \leq \sum_{v \in V(G-e)}\left|f^{\prime}(v)\right| \leq \sum_{v \in V(G)}|f(v)|+k-1=\gamma_{i_{r k}}(G)+k-1 .
$$

Case 2. If $f$ assigns $\varnothing$ to all vertices in $N_{G}(y)-\{x\}$, then $f^{\prime}: V(G-e) \rightarrow \mathcal{P}(\{1,2, \ldots, k\})$ defined by

$$
f^{\prime}(v)= \begin{cases}\{1\} & \text { if } v=y \\ f(v) & \text { if } v \neq y\end{cases}
$$

would be an $\mathrm{I} k$-rD function of $G-e$. Thus,

$$
\gamma_{i_{r k}}(G-e) \leq \sum_{v \in V(G-e)}\left|f^{\prime}(v)\right|=\sum_{v \in V(G)}|f(v)|+1=\gamma_{i_{r k}}(G)+1 .
$$

This ends the proof. 


\section{3-Rainbow and Weak $\{3\}$-Domination Numbers}

A linear algorithm for determining a 2-rD function of minimum weight of an arbitrary tree has been presented in [2]. The algorithm was based on the related concept of the so-called weak 2-domination. Intuitively, we could call it a monochromatic version of the 2-rainbow domination. Let $G=(V, E)$ be a graph and $f$ be a function from $V(G)$ to $\{0,1,2,3\}$. In this section, we want to establish related concept of the so-called weak 3-domination and 3-rainbow domination.

For $v \in V$, we define

$$
f[v]=\sum_{u \in N[v]} f(u)
$$

for notational convenience. We call a vertex $v \in V$ a bad vertex with respect to $f$ if $f(v)=0$ and $|f[v]| \leq 2$; otherwise, we say that $v$ is a good vertex with respect to $f$. Note that if $v$ is a good vertex with respect to $f$ and $f(v)=0$, then $|f[v]| \geq 3$. If every vertex of $G$ is a good vertex with respect to $f$, then $f$ is called a weak $\{3\}$-dominating function (W3D function) of $G$. The weight $w(f)$ of $f$ is defined as $w(f)=\sum_{v \in V} f(v)$. The minimum weight of a W3D function of $G$ is called the weak $\{3\}$-domination number of $G$, which we denote it by $\gamma_{w 3}(G)$.

The main reason for introducing this concept is the following.

Theorem 4.1. ( [2]) For every tree $T, \gamma_{r 2}(T)=\gamma_{w 2}(T)$.

Here we show that the corresponding result of Theorem 4.1 holds for $\gamma_{r 3}(T)$ and $\gamma_{w 3}(T)$.

Theorem 4.2. For any tree $T, \gamma_{r 3}(T)=\gamma_{w 3}(T)$.

Proof. Let $T=(V, E)$ and $g$ be a 3-rD function of $T$ of minimum weight. We define $f_{g}: V \longrightarrow\{0,1,2,3\}$ by $f_{g}(v)=$ $|g(v)|$, for all $v \in V$. Then $f_{g}$ is a W3D function of $T$ of weight $w\left(f_{g}\right)=w(g)=\gamma_{r 3}(T)$, and so $\gamma_{w 3}(G) \leq w\left(f_{g}\right)=\gamma_{r 3}(T)$.

It now suffices to show that $\gamma_{r 3}(T) \leq \gamma_{w 3}(T)$. Let $f$ be a $\gamma_{w 3}(T)$-function. Let $g_{f}: V \longrightarrow \mathcal{P}(\{1,2,3\})$ be defined as follows. If $f(v)=0$, let $g_{f}(v)=\varnothing$. If $f(v)=3$, let $g_{f}(v)=\{1,2,3\}$. If $f(v)=1(f(v)=2)$, let $g_{f}(v)$ be chosen so that

(i) $g_{f}(v)=\{1\},\{2\}$ or $\{3\}\left(g_{f}(v)=\{1,2\},\{1,3\}\right.$ or $\left.\{2,3\}\right)$, and

(ii) the number of vertices $v$ for which $g_{f}(v) \neq \varnothing$ or $\bigcup_{u \in N[v]} g_{f}(u)=\{1,2,3\}$ is maximum.

We show that then for every vertex $v \in V(G)$, we have $g_{f}(v) \neq \varnothing$ or $\bigcup_{u \in N[v]} g_{f}(u)=\{1,2,3\}$ (and therefore $g_{f}$ is a 3-rD function of $T$ ). Suppose to the contrary that, there exists a vertex $v$ not having this property with respect to $g_{f}$. Taking into account this fact and since $v$ is a good vertex with respect to $f$, we infer that $f(v)=0$, no neighbor of $v$ has weight 3 under $f$ and therefore all neighbors of $v$ have weights at most 2 under $f$. We now have three possible cases.

Case 1. There exist three vertices $x, y$ and $z$ in $N(v)$ with $f(x)=f(y)=f(z)=1$. In the worst case, we may assume that $g_{f}(x)=g_{f}(y)=g_{f}(z)=\{1\}$ (note that a similar argument will be held in the cases $g_{f}(x)=g_{f}(y)=g_{f}(z)=\{2\}$ or $\{3\})$. Let $T_{x}$ and $T_{y}$ be the components of $T-v$ containing $x$ and $y$, respectively. Let $g_{f}^{\prime}$ be obtained from $g_{f}$ by exchanging the roles of 1 and 2 on $V\left(T_{x}\right)$, and exchanging 1 and 3 on $V\left(T_{y}\right)$. Since $g_{f}^{\prime}(x)=\{2\}, g_{f}^{\prime}(y)=\{3\}$ and $g_{f}^{\prime}(z)=\{1\}$, we have $\bigcup_{u \in N[v]} g_{f}^{\prime}(u)=\{1,2,3\}$. This is contrary to our choice of $g_{f}$.

Case 2. There exist two vertices $x$ and $y$ in $N(v)$ with $f(x)=1$ and $f(y)=2$. Then, $g_{f}(x)=\{a\}$ and $g_{f}(y)=\{b, c\}$, in which $1 \leq a, b, c \leq 3$ and $a=b$. Let $d \in\{1,2,3\} \backslash\{b, c\}$. Let $g_{f}^{\prime \prime}$ be obtained from $g_{f}$ by exchanging the roles of $d$ and $a$ on $V\left(T_{x}\right)$. We now have $g_{f}^{\prime \prime}(x)=\{d\}$ and $g_{f}^{\prime \prime}(y)=\{b, c\}$, and thus $\bigcup_{u \in N[v]} g_{f}^{\prime \prime}(u)=\{1,2,3\}$. This is contrary to our choice of $g_{f}$.

Case 3. There exist two vertices $x$ and $y$ in $N(v)$ with $f(x)=f(y)=2$. Similar to the Case 2, we derive a contradiction.

The function $g_{f}$ would be a 3-rD function of $T$ with weight $\gamma_{w 3}(G)$. So, $\gamma_{r 3}(T) \leq \gamma_{w 3}(G)$.

If $U$ is a unicycle graph, then by deleting an edge from the cycle it will be changed to a tree. Thus we have the following proposition.

Proposition 4.3. For a unicycle graph $U, \gamma_{r 3}(U) \leq \gamma_{w 3}(U)$.

Proof. By Theorem 4.2 and Proposition 3.1 the result follows.

\section{Conflicts of INTEREST}

The authors declare that there are no conflicts of interest regarding the publication of this article. 


\section{REFERENCES}

[1] Amjadi, J., Dehgardi, N., Mohammadi, N., Sheikholeslami, S.M., Volkmann, L., Independent 2-rainbow domination numbers in trees, AsianEur. J. Math., 08(2015) 02, 1550035. 1, 2

[2] Bresar, B., Henning, M., Rall, D., Rainbow domination in graphs, Taiwanese J. Math. 12(2008), 23-225. 1, 1.1, 1, 4, 4.1

[3] Bresar, B., Sumnjak, T., On 2-rainbow domination in graphs, Discrete Appl. Math. 155(2007), 2394-2400. 1, $2,2.4$

[4] Chang, G., Wu, J., Zhu, X., Rainbow domination on trees, Discrete Appl. Math. 158(2010), 8-12. 1

[5] Hao, G., Mojdeh, D.A., Wei, S., Xie, Z., Rainbow domination in the Cartesian product of directed paths, Australas. J. Combin. 70(3)(2018), 349-361. 1

[6] Mansouri, Z., Mojdeh, D.A., Outer independent rainbow dominating functions in graphs, Opuscula Math. 40(5)(2020), 599-615 https://doi.org/10.7494/OpMath.2020.40.5.599. 1

[7] Mojdeh, D.A., Kazemi, A.P., Domination in Harary graphs, Bulletin of the Institute of Combinatorics and its Application 49(2007), 61-78. 2

[8] Pai, K., Chiu, W., 3-rainbow domination number in graphs, In Proceedings of the Institute of Industrial Engineers Asian Conference 2013, 713-720. Springer, Science+ Business Media Singapore, 2013. 1

[9] West, D.B., Introduction to Graph Theory (Second Edition) (Prentice Hall, USA, 2001). 1

[10] Wu, Y., Jafari Rad, N., Bounds on the 2-rainbow domination number of graphs, Graphs and Combin. 29(2013), 1125-1133. 1 